\title{
The embedded star clusters in the nebulae vdB-RN 92 and Gy 3-7 in Canis Majoris R1
}

\author{
J. B. Soares and E. Bica \\ Universidade Federal do Rio Grande do Sul, IF, CP 15051, Porto Alegre 91501-970, RS, Brazil \\ Received 23 December 2002 / Accepted 25 March 2003

\begin{abstract}
We employed $J, H$ and $K_{\mathrm{s}}$ photometry from the 2MASS Point Source Catalogue to study the embedded star clusters in the nebulae vdB-RN 92 and Gy 3-7, which are located in the molecular cloud Canis Majoris R1. We employed colourcolour and colour-magnitudes diagrams together with theoretical pre-main sequence isochrones to derive their fundamental parameters. Ages are based on the fraction of stars with anomalous colours, supposedly of types Herbig AeBe and T Tauri. The vdB-RN 92 cluster has an age of 5-7 Myr and the Gy 3-7 cluster $\approx 2 \mathrm{Myr}$. The average reddening values are $A_{V}=4.4$ and $A_{V}=6.3$, respectively for vdB-RN 92 and Gy 3-7. The distance found for vdB-RN 92 was $1.5 \mathrm{kpc}$ which is, within uncertainties, compatible with previous estimates for the complex. Gy 3-7 also appears to be related to the CMaR1 complex.
\end{abstract} \\ We conclude that CMaR1 is a very active star-forming site, now with four star clusters in early evolutionary stages.
}

Key words. Galaxy: open clusters and associations: individual: Gy 3-7, vdB-RN 92

\section{Introduction}

IR photometry is very useful to detect sites of star formation, in particular, young clusters in nebulae or molecular clouds. In these regions many studies have revealed the presence of embedded star clusters (e.g. Hodapp 1994; Carpenter et al. 1993). Soares \& Bica (2002) presented a study of two embedded star clusters in the molecular cloud complex CMaR1 using the Trapezium star cluster as a colour-magnitude diagram (CMD) template. The CMaR1 star forming region was first identified by van den Bergh (1966), as a group of stars embedded in reflection nebulosity. In the same area Clariá (1974a,b) identified an OB stellar association which appears to be a slightly evolved component of the star forming complex. Several Herbig AeBe stars occur in CMaR1. Clariá (1974b) and Shevchenko (1999) found distances of $\approx 1.15 \mathrm{kpc}$ and $\approx 1.05 \mathrm{kpc}$ to the association, respectively.

The CMaR1 molecular complex presents the possibility of other embedded star clusters or stellar groups. Thus it is important to further probe it with the tools of IR photometry. The reflexion nebula vdB-RN 92 was identified by van den Bergh (1966); it contains embedded stars, forming a small clustering as pointed out by himself. Gyulbudaghian (1984) identified a small nebula in CMaR1 - Gy 3-7, which was reported to contain a star cluster by Tapia et al. (1997).

According to the SIMBAD database (http://simbad. u-strasbg.fr) vdB-RN 92 includes the B1.5V star BD11 1763, as well as the infrared source IRAS 07016-1129. Tapia et al. (1997) report young stellar objects in the

Send offprint requests to: J. B. Soares, e-mail: jules@if.ufrgs.br range B1-B5 for Gy 3-7. The nebula also contains the infrared source IRAS 07069-1045.

The 2MASS (2 Micron All Sky Survey) Atlas and Point Source Catalogue are fundamental tools to probe the stellar content of star forming regions, and they are the result of an all-sky near infrared survey (Skrutskie et al. 1997). The effective wavelengths of the photometric bands are $1.25 \mu \mathrm{m}(J)$, $1.65 \mu \mathrm{m}(H)$ and $2.17 \mu \mathrm{m}\left(K_{\mathrm{s}}\right)$. The $K_{\mathrm{s}}$ band is thus located slightly shortwards of the $K$ band. The data (second incremental release) can be obtained in the web interface http://irsa.ipac.caltech.edu.

In the present study we analyse the stellar content of the nebulae vdB-RN 92 and Gy 3-7 by means of colour-colour and CMDs using the 2MASS Point Source Catalogue. In particular we verify the suggestion by van den Bergh of a clustering in the nebula vdB-RN 92. In Sect. 2 we describe the objects based on 2MASS and Digitized Sky Surveys imaging. In Sect. 3 we present the 2MASS photometric extractions in the bands $J H K_{\mathrm{s}}$. In Sect. 4 we discuss the analysis methods and the photometric diagrams of the embedded clusters in vdB-RN 92 and Gy 3-7. Finally, in Sect. 5 the concluding remarks of this work are given.

\section{The objects}

The clustering in vdB-RN92 is located at J2000.0 $\alpha=$ $7^{\mathrm{h}} 03^{\mathrm{m}} 56^{\mathrm{s}} \delta=-11^{\circ} 34^{\prime} 30^{\prime \prime}\left(l=224^{\circ} 65, b=-2.52\right)$. A 2MASS $K_{\mathrm{s}}$ band image of the region is presented in Fig. 1. A concentration of stars occurs within a radius of $\approx 1^{\prime}$.

The cluster or stellar group in Gy $3-7$ is located at $\alpha=$ $7^{\mathrm{h}} 09^{\mathrm{m}} 21^{\mathrm{s}} \delta=-10^{\circ} 50^{\prime} 30^{\prime \prime}\left(l=224^{\circ} .61, b=-1.00\right)$ and its 


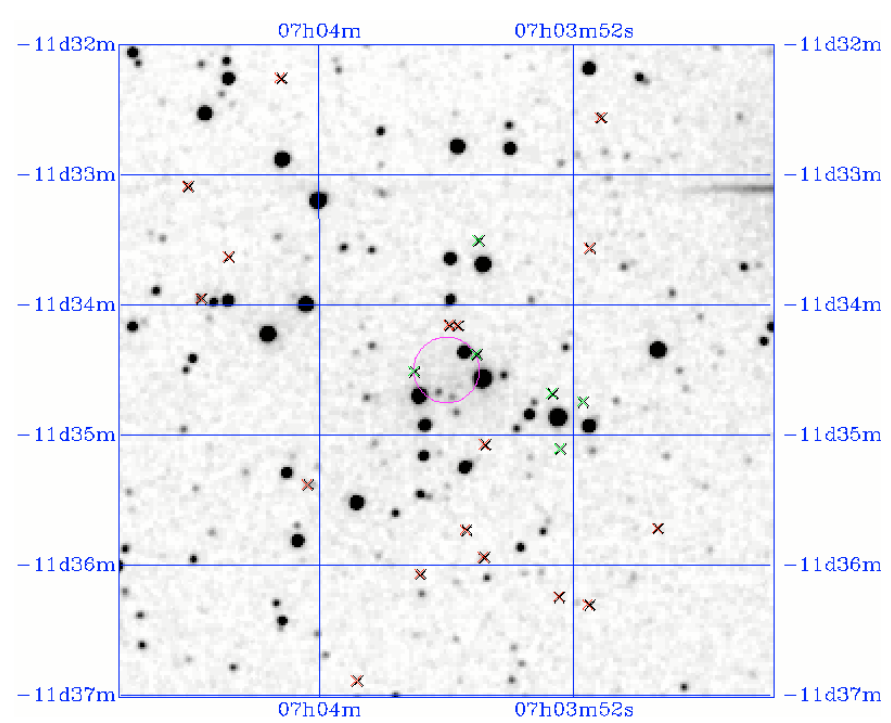

Fig. 1. $K_{\mathrm{s}}$ band 2MASS image of vdB-RN 92. Open circle indicates the object central part. Crosses indicate instrumental artifacts.

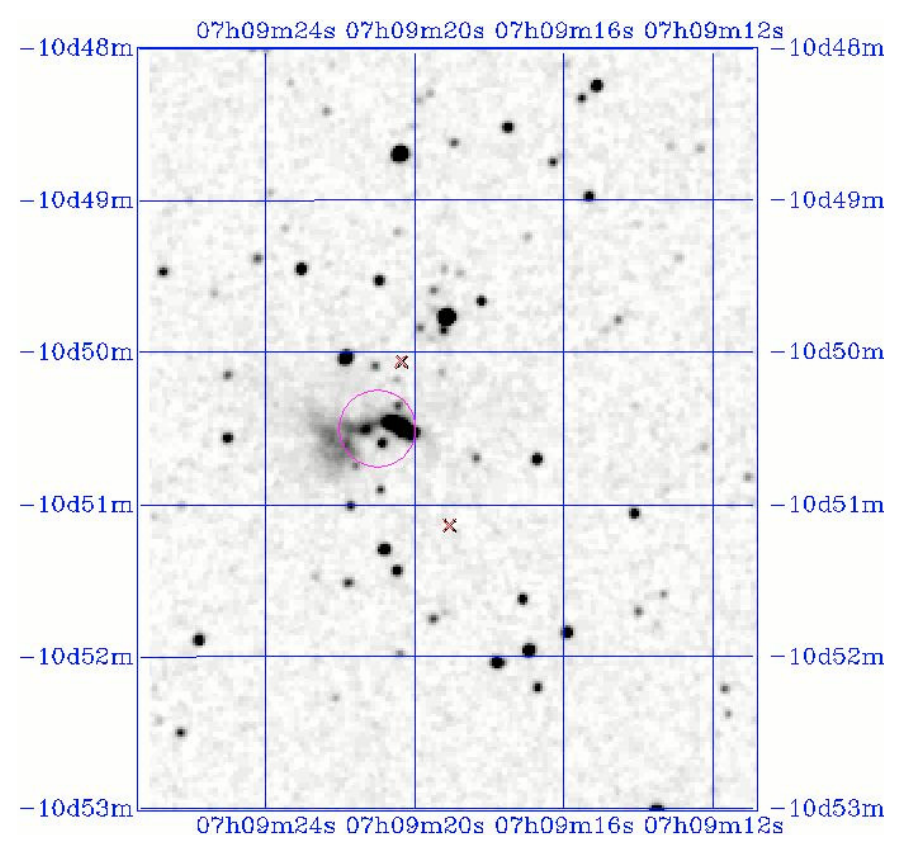

Fig. 2. Same as Fig. 1 for Gy 3-7.

angular radius is $\approx 1^{\prime}$. In Fig. 2 the $K_{\mathrm{s}}$ image shows concentration of stars and some nebular emission and/or reflexion.

$R$ band Digitized Sky Survey images http: //cadcwww . dao.nrc.ca/cadcbin/getdss of vdB-RN 92 and Gy 3-7 are given in Figs. 3 and 4, respectively. The vdB-RN 92 image shows a concentrated group of bright stars standing out in a diffuse dust dominated region. The nebulous star located at $\approx 3.4^{\prime} \mathrm{NW}$ of vdB-RN92 is the Herbig Be star ZCMa which has a bipolar outflow (Poetzel et al. 1989). In the Gy 3-7 image what stands out is the dust refletion and/or gas emission component. Extinction estimates in the directions of the two objects can be obtained from Schlegel et al. (1998): $A_{V}=9.5$ for vdB-RN 92 and $A_{V}=7.9$ for Gy 3-7. Owing to the expected strong extinction the stellar content of the two nebulae requires IR photometry for detailed studies.

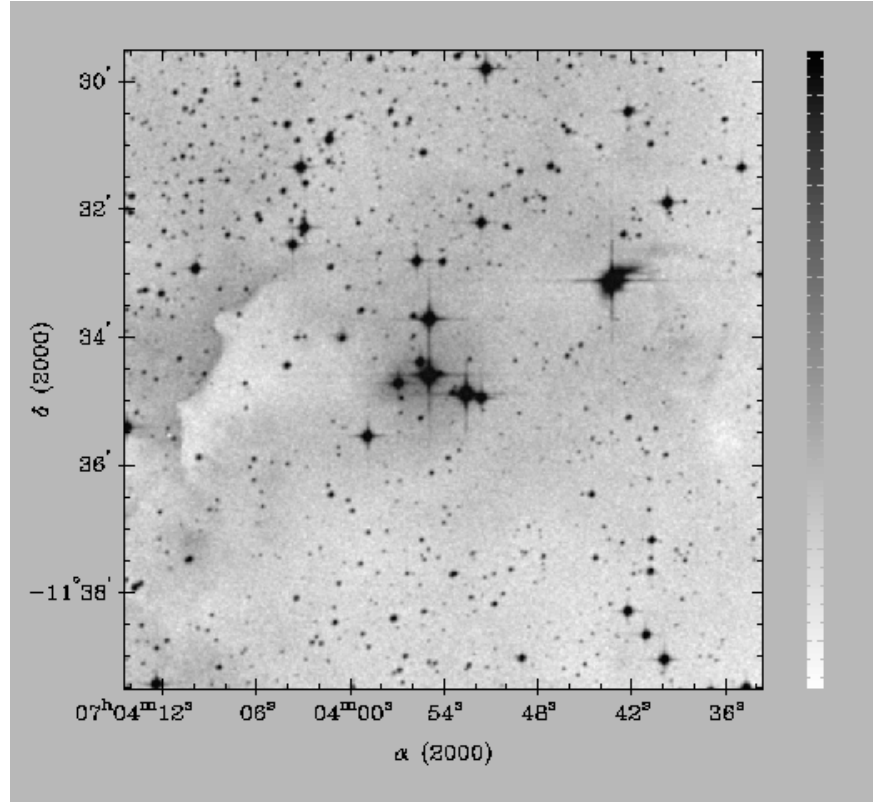

Fig. 3. $R$ image of vdB-RN 92 from the Digitized Sky Survey.

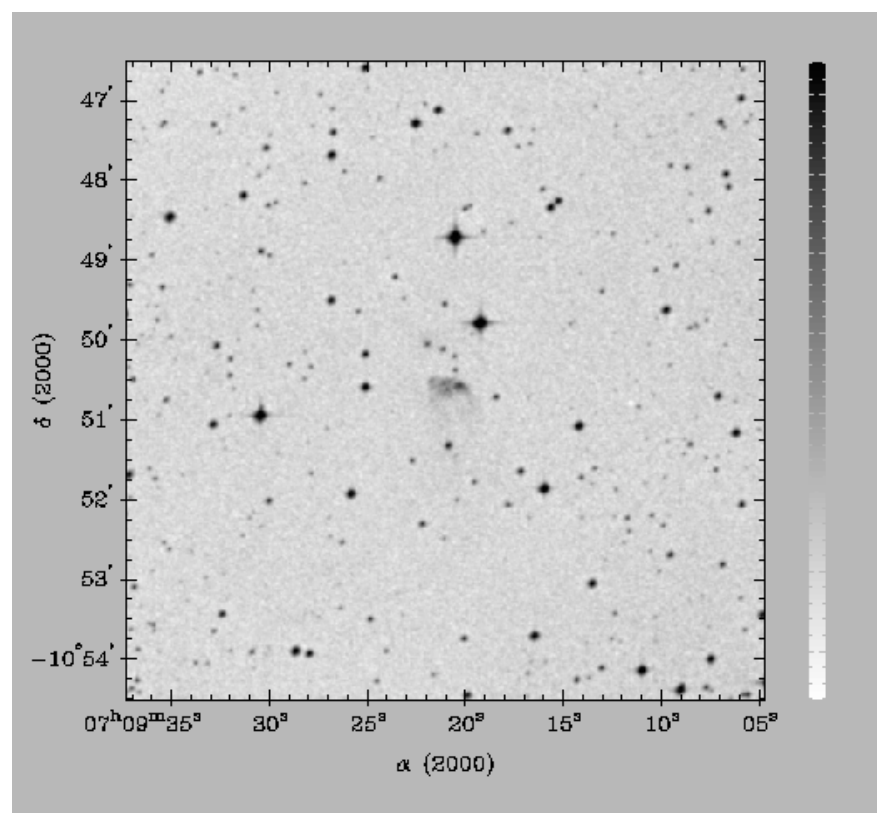

Fig. 4. Same as Fig. 3 for Gy 3-7.

In Fig. 5 is shown a $12 \mu \mathrm{m}$ band IRAS image of CMaR1. This band is particularly sensitive to warm dust, and in turn to the presence of star formation. vdB-RN 92 corresponds to a small blob in the main body of CMaR1. Gy 3-7 corresponds to the faint blob to the east and outside the main body. The overall cloud appears to have several cores, probably caused by shock fronts.

\section{IR photometry}

We employed the Gator tool for Point Source Catalogue extractions, as provided in the 2MASS site. An extraction table gives for each star besides the $J, H$ and $K_{\mathrm{s}}$ magnitudes, the three corresponding colours, the respective errors, and J2000.0 


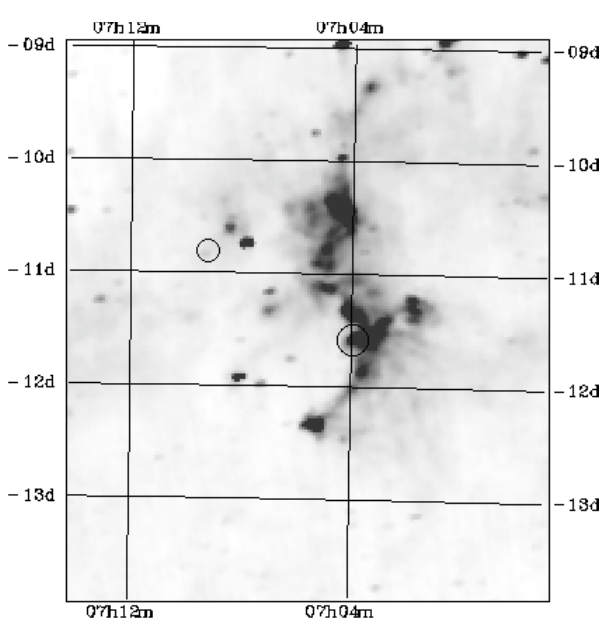

Fig. 5. IRAS $12 \mu \mathrm{m}$ image of CMaR1. Open circles encompass vdB-RN 92 (right) and Gy 3-7 (left).

coordinates. The 2MASS photometric errors in the present regions for each magnitude are basically the same as those presented in Soares \& Bica (2002) for the regions of NGC 2327 and BRC 27. Essentially, the errors become important (0.1 mag and larger) for magnitudes fainter than $K_{\mathrm{s}}=14$. We applied a cutoff at $K_{\mathrm{s}}=14.5$ for the stars to be studied in the colourcolour diagrams, for the sake of accuracy in their analyses. For comparison purposes we made extractions for the objects and their surrounding fields. Typical separations for objects and fields are $\approx 5^{\prime}$. Table 1 condenses information on the extractions: target position and number of extracted stars for radii $1^{\prime}$. Fields 1 to 4 are related to vdB-RN 92 and fields 5 to 8 to Gy 3-7. The numbers of stars in the cluster extractions exceed those of all fields.

\section{Discussion}

\subsection{The analysis methods}

The sensitivity increase of IR detectors provided new opportunities to observe and analyse the stellar content in molecular clouds. Regions of star formation contain many stars presenting IR excesses which are characteristic of very young objects. Colour-colour diagrams are a useful tool to deal with very young objects in the pre-main-sequence (PMS) stage (Carpenter et al. 1993). This method enables detections of $K_{\mathrm{s}}$ band excesses, which originate in dust disks or envelopes of contracting objects. Large reddening values are obtained in these regions as compared to surrounding field stars.

Both vdB-RN 92 and Gy 3-7 contain dust embedded stars as revealed by reflection nebulae in the optical/near-IR and warm dust emission in the infrared (Sect. 2), probably in the stellar formation phase.

The analysis employs colour-colour diagrams and CMDs. Finally, PMS isochrones by Siess et al. (2000) are used. The empirical isochrone for an age of 1.5 Myr derived from a template cluster (Trapezium) in Soares \& Bica (2002) turned out to be inappropriate for the present clusters, because they are in a later evolutionary stage.
Table 1. Position and number of stars for 2MASS extractions.

\begin{tabular}{lccc}
\hline \hline Name & $\alpha(\mathrm{J} 2000)$ & $\delta(\mathrm{J} 2000)$ & $n\left(r=1^{\prime}\right)$ \\
\hline vdB-RN 92 & $7^{\mathrm{h}} 03^{\mathrm{m}} 56^{\mathrm{s}}$ & $-11^{\circ} 34^{\prime} 30^{\prime \prime}$ & 31 \\
Field 1 & $7^{\mathrm{h}} 04^{\mathrm{m}} 45^{\mathrm{s}}$ & $-11^{\circ} 22^{\prime} 05^{\prime \prime}$ & 21 \\
Field 2 & $7^{\mathrm{h}} 03^{\mathrm{m}} 08^{\mathrm{s}}$ & $-11^{\circ} 25^{\prime} 36^{\prime \prime}$ & 26 \\
Field 3 & $7^{\mathrm{h}} 03^{\mathrm{m}} 13^{\mathrm{s}}$ & $-11^{\circ} 46^{\prime} 20^{\prime \prime}$ & 18 \\
Field 4 & $7^{\mathrm{h}} 04^{\mathrm{m}} 35^{\mathrm{s}}$ & $-11^{\circ} 45^{\prime} 21^{\prime \prime}$ & 21 \\
Gy 3-7 & $7^{\mathrm{h}} 09^{\mathrm{m}} 21^{\mathrm{s}}$ & $-10^{\circ} 50^{\prime} 30^{\prime \prime}$ & 33 \\
Field 5 & $7^{\mathrm{h}} 08^{\mathrm{m}} 55^{\mathrm{s}}$ & $-10^{\circ} 59^{\prime} 00^{\prime \prime}$ & 14 \\
Field 6 & $7^{\mathrm{h}} 09^{\mathrm{m}} 45^{\mathrm{s}}$ & $-10^{\circ} 43^{\prime} 00^{\prime \prime}$ & 19 \\
Field 7 & $7^{\mathrm{h}} 09^{\mathrm{m}} 48^{\mathrm{s}}$ & $-11^{\circ} 00^{\prime} 00^{\prime \prime}$ & 17 \\
Field 8 & $7^{\mathrm{h}} 08^{\mathrm{m}} 47^{\mathrm{s}}$ & $-10^{\circ} 40^{\prime} 12^{\prime \prime}$ & 17 \\
\hline
\end{tabular}

Stars at formation stages (T Tauri and Herbig AeBe) can exhibit anomalous colours due the $K_{\mathrm{s}}$ band excesses created by the hot dust emission around them (Lada \& Adams 1992; Calvet et al. 1992). In the $\left((J-H),\left(H-K_{\mathrm{s}}\right)\right)$ colour-colour diagram we can diagnose this effect. By means of the percentage of anomalous stars we can estimate the cluster or stellar group age, as described Lada et al. (1996). This diagram can also give the mean reddening of the object. In the present analysis, stars with anomalous colours were discarded from the calculation of the mean reddening.

\subsection{The cluster in the nebula $v d B-R N 92$}

In Fig. 6 we show $J,(J-H)$ CMDs of the cluster and the comparison fields 1 and 3 (Table 1). The cluster is more reddened than field 1 and slightly more reddened than field 3 .

The $\left((J-H),\left(H-K_{\mathrm{s}}\right)\right)$ colour-colour diagram is shown in Fig. 7. The continuous curve represents the intrinsic colours of main-sequence (MS) stars with spectral types from O3 to M5 (Schmidt-Kaler 1982). The dashed line denotes the reddened loci (Rieke \& Lebofsky 1985) for a MS O3 star, while the point-dashed line is for an M5 giant. The arrow indicates the extinction vector for $A_{V}=10$. We applied a 2MASS sample cutoff at $K_{\mathrm{s}}=14.5$ in order to avoid errors larger than $0.1 \mathrm{mag}$. The figure shows a number of stars distributed along the reddening vector, and some are little reddened and coincident with the field locus. The mean reddening is $A_{V}=4.4$ considering only the stars along the reddening vector. This reddening value corresponds to $A_{J}=1.21$ and $E(J-H)=0.47$. The reddening transformations employed were derived from the data in Rieke \& Lebofsky (1985) assuming $A_{V}=3.2 E(B-V)$. Some stars have $\left(H-K_{\mathrm{s}}\right)$ colours denoting infrared excess (below the dashed line). The vdB-RN 92 cluster has $\approx 20 \%$ of stars with infrared excess, similarly to the IC 348 cluster (Lada \& Lada 1995) implying an age of 5-7 Myr.

In the CMD analysis of the vdB-RN92 cluster PMS isochrones of Siess et al. (2000) were used. These authors present isochrone grids for the range 0.1 to $7.0 M_{\odot}$ and four different metallicities $(Z=0.001,0.02,0.03$ and 0.04$)$. For the present analyses we adopted the sollar metallicity isochrones. For more massive stars we include the Padova isochrone of 4 Myr (Bertelli et al. 1994) as complement to the Zero Age 


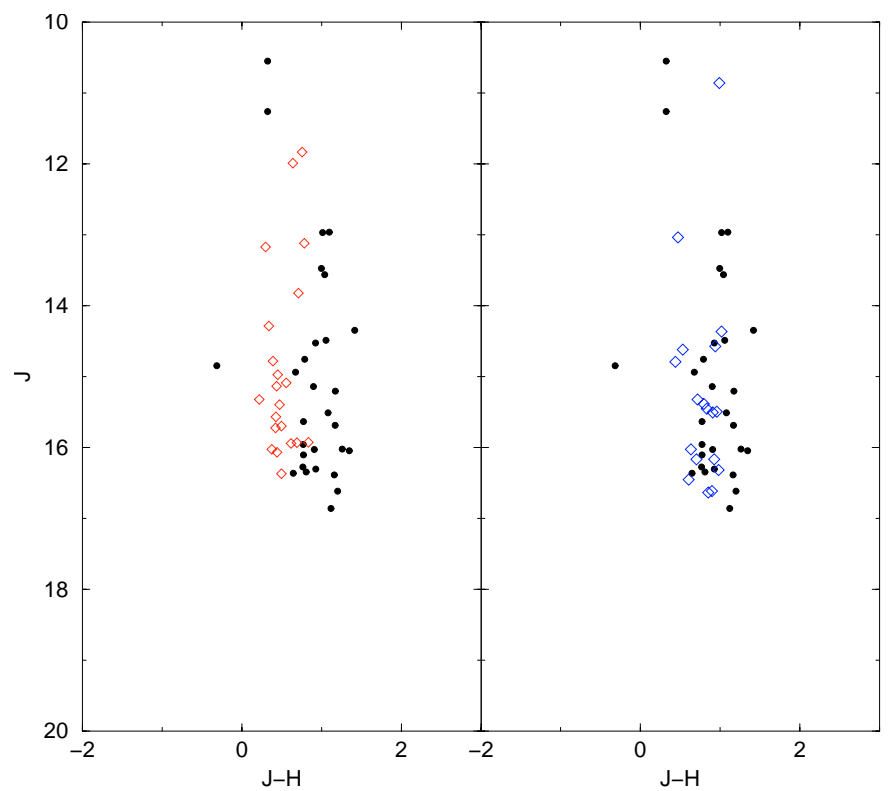

Fig. 6. vdB-RN 92: $J,(J-H)$ CMD of the cluster region (filled circles) and comparison fields (open diamonds). Field 1 corresponds to the left panel and field 3 to the right one. All extractions have radius $1^{\prime}$. No cutoff was used.

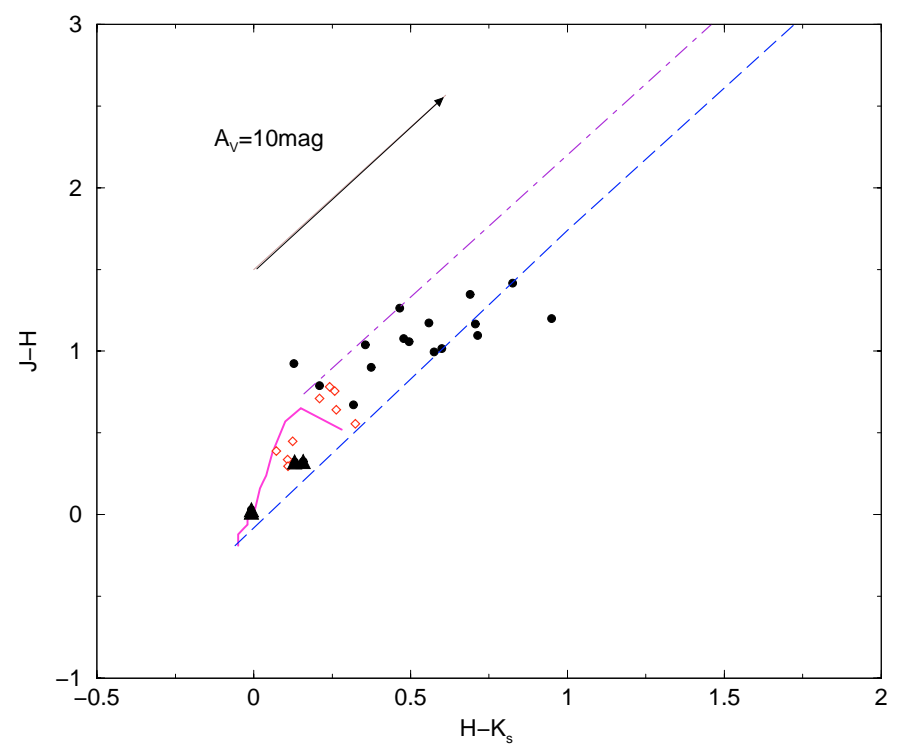

Fig. 7. vdB-RN 92: $\left((J-H),\left(H-K_{\mathrm{s}}\right)\right)$ diagram for the cluster (filled circles) and comparison field 1 (open diamonds). Triangles are the four more luminous stars in the object. Reddening vector and reddening lines for M5 giant (dashed-pointed line) and O3 stars (dashed line) are shown. The continuous curve is the intrinsic distribution of spectral types. A cutoff at $K_{\mathrm{s}}=14.5$ was used.

Main-Sequence (ZAMS) of Siess et al. (2000). The method of fraction of stars with infrared excess to determine ages is more appropriate than that of the isochrones, because differential reddening and age dispersion within the cluster hamper its application. Spectroscopic observations of the brightest star in vdB-RN92 indicated spectral type B1.5 (Herbst et al. 1978). The colours and magnitudes of a B1.5 MS star (Schmidt-Kaler 1982) were used as constraint to the fit (Fig. 8 - left panel). The observed distance modulus is $J-M_{J}=11.16$

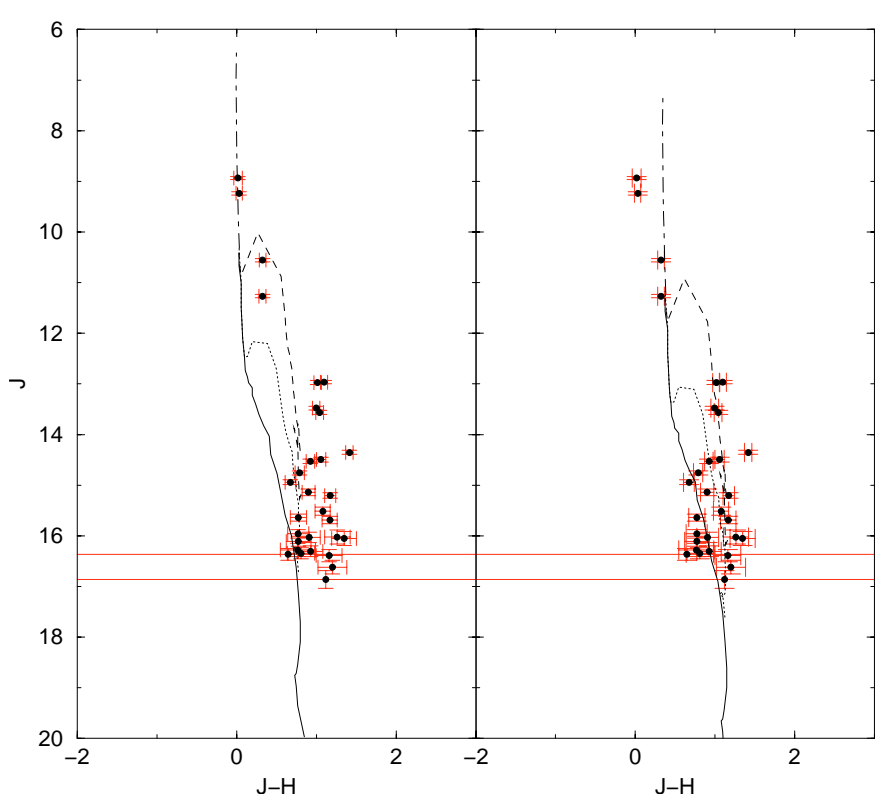

Fig. 8. vdB-RN 92: $J,(J-H)$ CMD of the cluster (filled circles) with PMS isochrones of $1 \mathrm{Myr}$ (dashed line) and $7 \mathrm{Myr}$ (dotted line). The ZAMS is represented by continuous line (Siess et al. 2000) and dashed-dotted line (Bertelli et al. 1994). Left panel presents the fit for the most massive star. Right panel presents the fit with $A_{J}=1.21$. Colour and magnitude errors are indicated.

and $E(J-H)=0.11$. The distance from the Sun found for vdB-RN 92 is $d_{\odot}=1.5 \pm 0.3 \mathrm{kpc}$. Low mass stars cannot be described by the fit for the bright stars, suggesting differential reddening. This effect might occur due to a lower reddening in the line of sight of massive stars. In the colour-colour diagram (Fig. 7), the four brighter stars are near the locus of unreddened stars, while the faint ones are spread along the reddening lines. Between the deeply embedded phases within dust/molecular gas and an evolved cluster the interstellar material undergoes processes that certainly lead to non-isotropic dust distribution. These processes depend on the involved stellar masses. Lowmass protostars evolve much slower, release less radiative energy and drive less energetic winds (Boogert et al. 2000). The mean reddening found in the colour-colour diagram analysis was applied to the cluster CMD and the results are shown in Fig. 8 (right panel).

\subsection{The cluster in the nebula Gy 3-7}

In Fig. 9 we show the $J,(J-H)$ CMD for an extraction of radius $1^{\prime}$, centered on Gy 3-7. We show equal size extractions for the comparison fields 5 and 6 (Table 1). Redder colours occur for the stars in the cluster region, suggesting larger reddening. Note also the stronger reddening and differential reddening in Gy 3-7 as compared to vdB-RN 92 (Fig. 6).

Figure 10 shows a $\left((J-H),\left(H-K_{\mathrm{s}}\right)\right)$ diagram for the Gy 3-7 cluster, compared to that of field 5. A significant number of stars in the cluster present infrared excess $\left(H-K_{\mathrm{s}}>1-\right.$ or below the dashed line). The remaining cluster stars present larger reddening values than those of the field. 


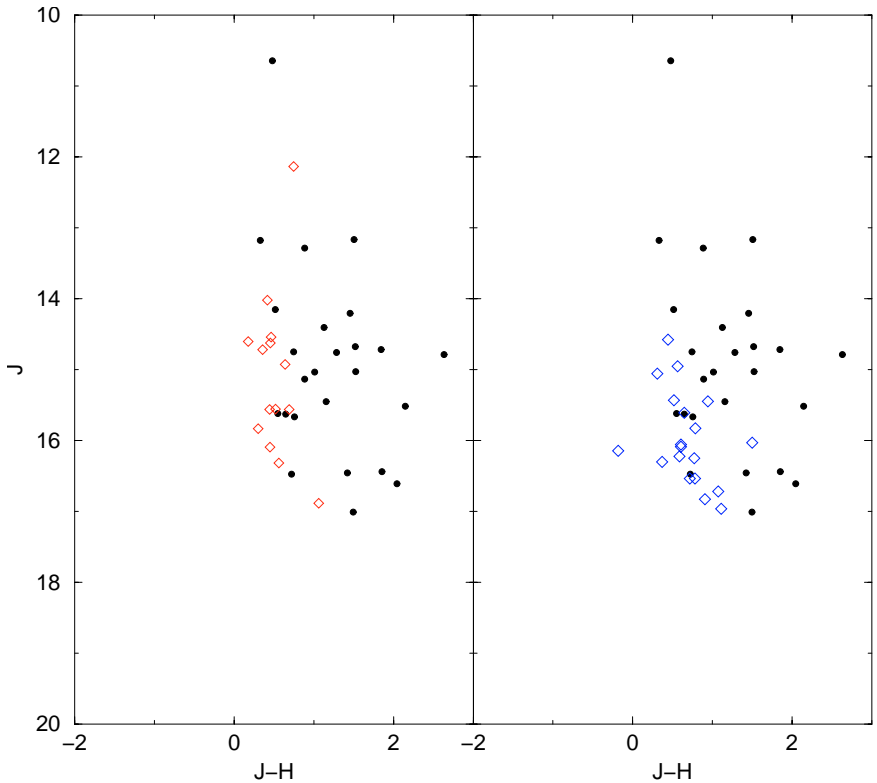

Fig. 9. Gy 3-7: $J,(J-H)$ CMD of the cluster region (filled circles) and comparison star fields (open diamonds). Field 5 corresponds to the left panel and field 6 to the right one. All extractions have radius $1^{\prime}$. No magnitude cutoff was used.

The mean reddening value resulted $A_{V}=6.3$, which implies $A_{J}=1.74$ and $E(J-H)=0.67$. For this estimate we excluded the stars with infrared excess.

The Gy 3-7 cluster has $\approx 40 \%$ of its stars with infrared excess, a percentage slightly lower than that in the Taurus Dark Nebula (Kenyon \& Hartmann 1995), suggesting an age of $\approx 2$ Myr. For this calculation we minimized field contamination by discarding the stars with extinction similar to those of field stars.

In the literature two distances have been proposed to Gy 3-7. Wouterloot \& Brand (1989) reported a kinematic distance of $1.41 \mathrm{kpc}$ from $\mathrm{CO}$ observations. This distance would imply an association to CMaR1, with estimated distances in the range 1.05-1.2 kpc (Shevchenko 1999; Soares \& Bica 2002). Tapia et al. (1997) suggested at least a factor two larger to explain ionizition in the nebula.

In Fig. 11 (left panel) we show a PMS fit to Gy 3-7 assuming a distance of $1.4 \mathrm{kpc}$ (Wouterloot \& Brand 1989) and a reddening $E(J-H)=0.27$ which is appropriate to the brightest star. The latter star is located near the locus of unreddened stars (Fig. 10). We conclude that this star is possibly a PMS around B5, hot enough $(T \approx 14300 \mathrm{~K}$, Siess et al. 2000) to provide ionization to the nebula, without the need of a larger distance as given in Tapia et al. (1997). In Fig. 11 (right panel) we show the same plot of Fig. 11a but with a larger reddening $E(J-H)=0.67\left(A_{J}=1.74\right.$ or $\left.A_{V}=6.3\right)$ which is appropriate to the lower mass stars. Differential reddening is important and several stars appear to have large $(J-H)$ excesses denoting dust envelopes.

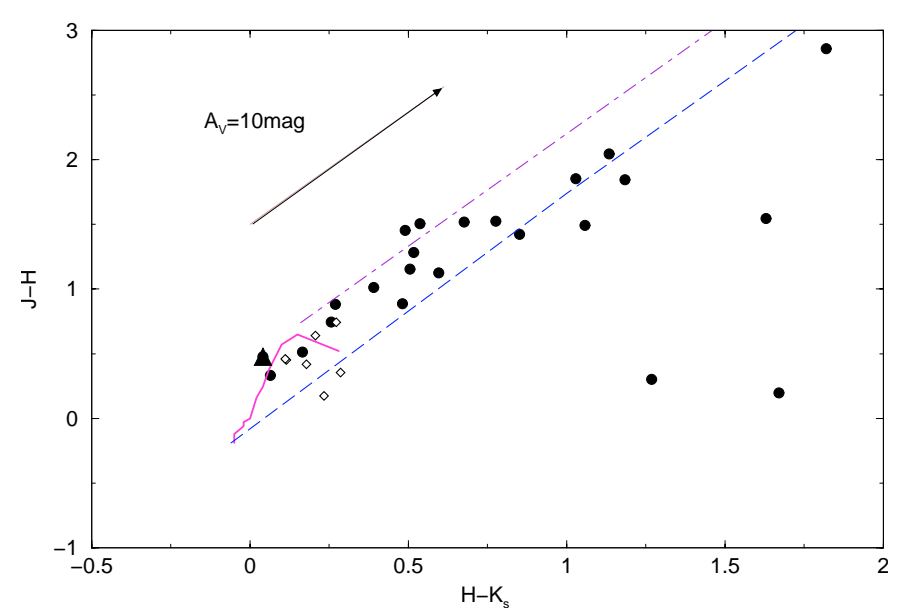

Fig. 10. Gy 3-7: $\left((J-H),\left(H-K_{\mathrm{s}}\right)\right)$ diagram for the cluster (filled circles) and comparison field 5 (open diamonds). Triangle is the most luminous star in the object. Reference lines as in Fig. 7. A cutoff at $K_{\mathrm{s}}=14.5$ was applied to minimize errors.

\section{Concluding remarks}

Two embedded star clusters in CMaR1 were studied by means of 2MASS $J$, Hand $K_{\mathrm{s}}$ photometry in the nebulae vdB-RN 92 and Gy 3-7. They are not massive clusters and there remains the possibility of classifying them as star clusters or stellar groups, in the sense of scarcely populated physical systems.

The vdB-RN92 cluster $(J-H),\left(H-K_{\mathrm{s}}\right)$ diagram presents $\approx 20 \%$ of its stars with infrared excess, suggesting an age of 5-7 Myr similar to that of the IC 348 cluster (Lada \& Lada 1995). The mean visible absorption is $A_{V}=4.4$. The estimated distance was $d_{\odot} \approx 1.5 \mathrm{kpc}$, where we used premain sequence isochrones and the most luminous star (B1.5) as constraint.

The Gy 3-7 cluster has $\approx 40 \%$ of its stars with infrared excess, implying an age of $\approx 2 \mathrm{Myr}$, likewise the Taurus Dark Nebula (Kenyon \& Hartmann 1995). The mean visible absorption in Gy 3-7 is $A_{V}=6.3$, as derived from the colour-colour diagram. The kinematic distance of $1.4 \mathrm{kpc}$ (Wouterloot \& Brand 1989) appears to be compatible with that of the cluster, where the most luminous star (B5) is in the pre-main sequence phase, and hot enough to ionize the gas.

The results indicate that the vdB-RN 92 cluster and the Gy 3-7 cluster are in distinct evolutionary stages. In both clusters there is evidence that the more massive stars are less reddened than low mass stars. This effect might be related to the way which stars of different masses affect the surrounding interstellar medium.

The CMaR1 cloud appears to be a very active site of starformation. In addition to the overall formation in the association (Shevchenko 1999) there occur enhanced sites. These are the present two clusters and those in the nebulae NGC 2327 and BRC 27 (Soares \& Bica 2002).

Acknowledgements. This publication makes use of data from the 2 Micron All Sky Survey, which is a joint project of the University of Massachusetts and the Infrared Processing and Analysis Center, funded by the National Aeronautics and Space Administration and the National Science Foundation. We employed data from the 


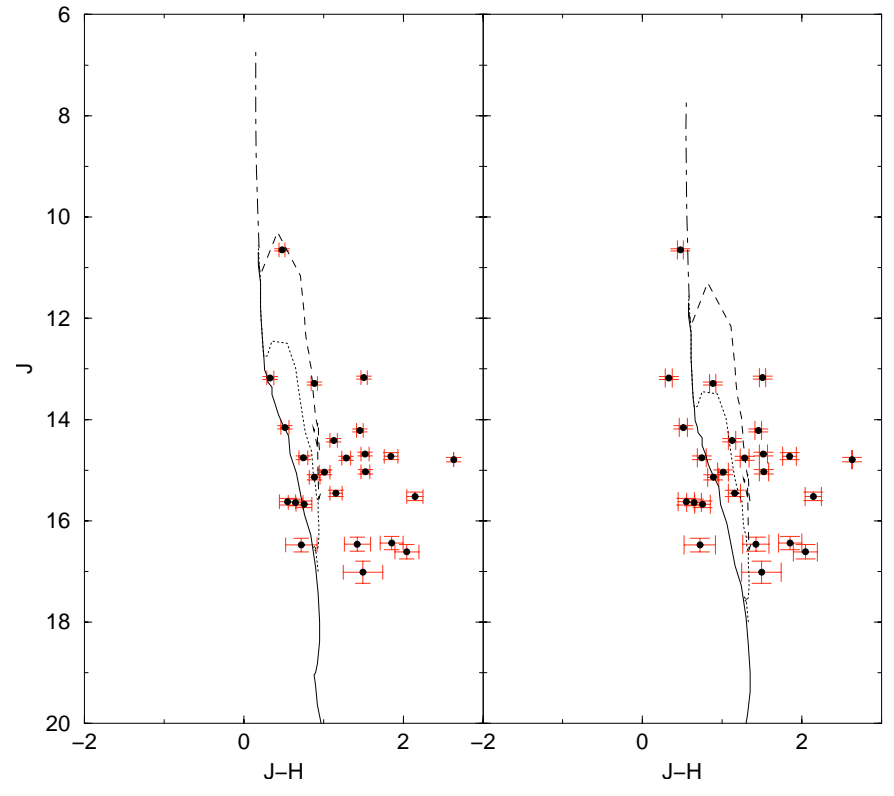

Fig. 11. Gy 3-7: $J,(J-H)$ CMD of the cluster (filled circles) with PMS isochrones of $1 \mathrm{Myr}$ (dashed line) and $7 \mathrm{Myr}$ (pointed line). The ZAMS is represented by continuous line (Siess et al. 2000) and dashed-dotted line (Bertelli et al. 1994). Left panel presents the fit for the distance found by Wouterloot \& Brand (1989). Right panel presents the fit with $A_{J}=1.74$. Colour and magnitude errors are indicated.

CDS database (Strasbourg). We also acknowledge support from the Brazilian Institutions CNPq and CAPES, in particular J.S. for a CNPq Ph.D. fellowship.

\section{References}

Bertelli, G., Bressan, A., Chiosi, C., Fagotto, \& F., Nasi 1994, A\&AS, 106, 275

Boogert, A. C. A., Tielens, A. G. G. M., Ceccarelli, C., et al. 2000, A\&A, 360, 683

Calvet, N., Magris, G. C., \& Patino, A. 1992, RMxAA, 24, 27

Carpenter, J. M., Snell, R. L., Schloerb, F. P., \& Skrutskie, M. F. 1993, ApJ, 407, 657

Clariá, J. J. 1974a, ApJ, 79, 1022

Clariá, J. J. 1974b, A\&A, 37, 229

Gyulbudaghian, A. L. 1984, Afz, 20, 631

Herbst, W., Racine, R., \& Warner, J. W. 1978, ApJ, 223, 471

Hodapp, W. K. 1994, ApJ, 94, 615

Kenyon, S. J., \& Hartmann, L. 1995, ApJS, 101, 117

Lada, C. J., \& Adams, F. 1992, ApJ, 393, 278

Lada, E. A., \& Lada, C. J. 1995, AJ, 109, 1682

Lada, C. J., Alves, J., \& Lada, E. A. 1996, AJ, 111, 1964

Poetzel, R., Mundt, R., \& Ray, T. P. 1989, A\&A, 224, L13

Rieke, G. H., \& Lebofsky, M. J. 1985, ApJ, 288, 618

Schlegel, D. J., Finkbeiner, D. P., \& Davis, M. 1998, ApJ, 500, 525

Schmidt-Kaler, T. 1982, in Landolt-Börnstein, New Ser., Group VI, vol. 2b (Springer-Verlag, Berlin), 1

Shevchenko, V. S. 1999, MNRAS, 310, 210

Siess, L., Dufour, E., \& Forestini, M. 2000, A\&A, 358, 593

Skrutskie, M., Schneider, S. E., Stiening, R., et al. 1997, In The Impact of Large Scale Near-IR Sky Surveys, ed. F. Garzon, N. Epchtein, A. Omont, W. B. Burton, \& P. Persi (Dordrecht: Kluwer), 25

Soares, J. B., \& Bica, E. 2002, A\&A, 388, 172

Tapia, M., Persi, P., Bohigas, J., \& Ferrari-Toniolo, M. 1997, AJ, 113, 1769

van den Bergh, S. 1966, AJ, 71, 990

Wouterloot, J. G. A., \& Brand, J. 1989, A\&AS, 80, 149 